BULLETIN OF THE

AMERICAN MATHEMATICAL SOCIETY

Volume 77, Number 4, July 1971

\title{
CHARACTERIZATIONS OF BOUNDED MEAN OSCILLATION
}

\author{
BY CHARLES FEFFERMAN
}

Communicated by M. H. Protter, December 14, 1970

BMO (bounded mean oscillation) is the Banach space of all functions $f \in L_{\text {loc }}^{1}\left(R^{n}\right)$ for which

$$
\|f\|_{\text {вMO }}=\sup _{Q}\left(\frac{1}{|Q|} \int_{Q}\left|f(x)-\operatorname{av}_{Q} f\right| d x\right)<\infty,
$$

where the sup ranges over all cubes $Q \subseteq R^{n}$, and $\operatorname{av}_{Q} f$ is the mean of $f$ over $Q$. See [5]. For convenience, we identify $f$ and $f^{\prime}$ in BMO if $f-f^{\prime}$ is constant.

TheOREM 1. BMO is the dual of the Hardy space $H^{1}\left(R^{n}\right)$. The inner product is given by $\langle f, g\rangle=\int_{R^{n}} f(x) g(x) d x$ for $f \in B M O$ and $g$ belonging to the dense subspace of $C^{\infty}$ rapidly decreasing functions in $H^{1}$.

Here, we regard $H^{1}$ as the space of $f \in L^{1}\left(R^{n}\right)$ whose Riesz transforms $R_{j}(f)$ are all in $L^{1}$. See [7].

THEOREM 2. A function belongs to BMO if and only if it can be written in the form $g_{0}+\sum_{j=1}^{n} R_{j}\left(g_{j}\right)$ with $g_{0}, g_{1}, \cdots, g_{n} \in L^{\infty}\left(R^{n}\right)$.

Note that the usual definition

$$
R_{j}(g)(x)=\lim _{\epsilon \rightarrow 0 ; M \rightarrow \infty} \int_{\epsilon<|x-y|<M} K_{j}(x-y) f(y) d y
$$

with $K_{j}(y)=c y_{j} /|y|^{n+1}$ need not make sense for all $g \in L^{\infty}$. (Consider $g(x)=\operatorname{sgn}(x)$ on the line.) Therefore, we define

$$
R_{j}(g)(x)=\lim _{\epsilon \rightarrow 0} \int_{\epsilon<|x-y|}\left[K_{j}(x-y)-K_{j}^{0}(-y)\right] g(y) d y,
$$

where $K_{j}^{0}(y)=K_{j}(y)$ for $|y|>1$ and $K_{j}^{0}(y)=0$ for $|y| \leqq 1$. This makes sense for all $g \in L^{\infty}$, and agrees with the usual definition up to an additive constant if $g$ has compact support. See [3, p. 105].

The main idea in proving Theorems 1 and 2 is to study the Poisson integral of a function in BMO. Recall that any function $f$ satisfying

AMS 1969 subject classifications. Primary 3067, 4635.

Key words and phrases. Bounded mean oscillation, Riesz transforms, maximal function, Poisson integral. 


$$
\int_{R^{n}} \frac{|f(x)|}{(|x|+1)^{n+1}} d x<\infty
$$

has a Poisson integral $u(x, t)=$ P.I. $(f)$ defined on $R_{+}^{n+1}=R^{n} \times(0, \infty)$.

THEOREM 3. A function $f$ belongs to BMO if and only if (*) holds and $\iint\left|x-x_{0}\right|<\delta ; 0<t<\delta t|\nabla u(x, t)|^{2} d x d t \leqq C \delta^{n}$ for all $x_{0} \in R^{n}$ and $\delta>0$.

Theorems 1-3 and their proofs can be used to study $H^{1}$. For example,

TheOREM 4. Let $F=\left(u_{0}(x, t) ; u_{1}(x, t), \cdots, u_{n}(x, t)\right)$ be an $(n+1)$ tuple of harmonic functions on $R_{+}^{n+1}$, satisfying the Cauchy-Riemann equations of [7]. If the nontangential maximal function $u_{0}^{*}(x)$ $\equiv \sup _{\left|x^{\prime}\right|<t ; t>0}\left|u_{0}\left(x-x^{\prime}, t\right)\right|$ belongs to $L^{1}$, then $F$ is in $H^{1}$.

Different techniques enable us to replace $L^{1}$ and $H^{1}$ by $L^{p}$ and $H^{p}$, $0<p<\infty$. This generalizes a one-dimensional result of D. Burkholder, R. Gundy, and M. Silverstein (see [1] and [2]).

Further applications of Theorems 1-3 appear in [4] and [6]. [4] contains detailed proofs of the results stated here.

\section{REFERENCES}

1. D. Burkholder and R. Gundy, Extrapolation and interpolation of quasi-linear operators on martingales, Acta Math. 124 (1970), 249-304.

2. D. Burkholder, R. Gundy and M. Silverstein, A maximal function characterization of the class $H^{p}$ (to appear).

3. A. P. Calderón and A. Zygmund, On the existence of certain singular integrals, Acta Math. 88 (1952), 85-139. MR 14, 637.

4. C. Fefferman and E. M. Stein, (in prep.)

5. F. John and L. Nirenberg, On functions of bounded mean oscillation, Comm. Pure Appl. Math. 14 (1961), 785-799.

6. E. M. Stein, $L^{p}$ boundedness of certain convolution operators, Bull. Amer. Math. Soc. 77 (1971), 404-405.

7. E. M. Stein and G. Weiss, Introduction to Fourier analysis on euclidean spaces, Princeton, 1971.

University of Chicago, Chicago, Illinois 60637 